\begin{tabular}{|c|l|}
\hline Title & Finite wavelength effect on the ball istic propagation of surface acoustic waves \\
\hline Author(s) & Tamura, Shin-ichiro; Y agi, Masashi \\
\hline Citation & $\begin{array}{l}\text { Physical Review B, 49(24), 17378.17384 } \\
\text { https://doi.org/40.1103/PhysRevB.49.17378 }\end{array}$ \\
\hline Issue Date & 199406-15 \\
\hline Doc URL & http://hdl.handle.net/2115/5957 \\
\hline Rights & Copyright $\odot$ 1994 A merican Physical Society \\
\hline Type & article \\
\hline File Information & PRB49-24.pdf \\
\hline
\end{tabular}

Instructions for use 


\title{
Finite-wavelength effect on the ballistic propagation of surface acoustic waves
}

\author{
Shin-ichiro Tamura and Masashi Yagi \\ Department of Engineering Science, Hokkaido University, Sapporo 060, Japan
}

(Received 29 December 1993)

\begin{abstract}
We study the phonon-focusing effects of the Rayleigh and pseudosurface waves propagating ballistically on the (100) surface of cubic crystals. Specifically, solving the wave equations for lattice displacements, we consider the finite-wavelength effect at the $\mathrm{MHz}$-frequency range and for 1-cm propagation distance. The amplitude and polarization of lattice displacements at the surface associated with surface waves are sensitive to the propagation direction. Owing to this characteristic of surface waves, the focusing factor defined in the ray picture does not necessarily describe correctly the intensity of surface waves excited from a point source. The angular dependences of the calculated displacement amplitudes explain an important feature of the recent focusing experiment of Rayleigh waves, which the simple theory based on the focusing factor fails to predict.
\end{abstract}

\section{INTRODUCTION}

Ballistic-phonon propagation in crystalline solids is profoundly influenced by the anisotropy of the lattices. The energy flux of phonons radiated from a small source exhibits huge anisotropy called phonon focusing. ${ }^{1,2}$ This effect arises from the fact that the group velocity and wave vector of a phonon are noncollinear, in general, due to crystal anisotropy. Experimentally, the phonon imaging technique has contributed substantially to the understanding of the phonon focusing effect in the bulk of solids. ${ }^{2}$

So far, phonon-focusing experiments have utilized high-frequency phonons of several hundred-GHz range and the observed focusing patterns coincide well with the ones calculated from the elasticity theory or lattice dynamical models based on the geometrical acoustics approximation, or ray picture for phonons. The validity of this approximation stems from the fact that the wavelength of a $100-\mathrm{GHz}$ phonon is about $300-500 \AA$ and the typical sizes of crystals used are several millimeters. Thus, the effect of the finite wavelength of phonons involved can be neglected in these experiments, validating the ray picture for phonons.

The effect of finite phonon wavelength on the phonon focusing was first studied by Maris. ${ }^{3}$ He found that the correction to the geometrical acoustics theory is enhanced near the caustic directions (where the geometrical acoustics approximation predicts infinitely large intensity) and proposed a suitably designed experiment to observe the effect for phonons of $100-\mathrm{GHz}$ frequency range.

Recently, experiments on the bulk-wave propagation and the related focusing with ultrasound beams have been made by several groups. ${ }^{4,5}$ Specifically, utilizing the ultrasonic imaging technique, Hauser, Weaver, and Wolfe $^{5}$ have observed very clearly the finite-wavelength effect on the phonon focusing, that is, the fringe patterns of acoustic intensity have been seen in the expected focusing regions. These patterns arise from the interfer- ence between waves with different wave vectors but with group velocities pointing in the same direction. It should be noted that in the strong focusing directions of phonons the group velocity surfaces (wave surfaces) are multivalued. The frequencies of the ultrasound used in the experiments are $5-20 \mathrm{MHz}$ and the corresponding wavelengths 1-0.25 $\mathrm{mm}$ are macroscopic. Thus, at these frequencies (and also for a sample thickness of the order of $1 \mathrm{~cm}$ ) the phonon-focusing caustics are found to evolve into a set of parallel fringes of the acoustic flux. This effect is called "internal diffraction" of acoustic waves in crystals. ${ }^{5}$

The focusing effect is also expected to occur for phonons propagating along crystal surfaces, i.e., surface phonons or surface acoustic waves. Theoretically, the focusing of Rayleigh surface waves (RSW) on the (100), (110), and (111) faces of GaAs and in several other crystals with lower symmetries has been studied by Tamura and Honjo. ${ }^{6}$ They used the ray approach and calculated the focusing factor $A=\left|\Delta \theta_{k} / \Delta \theta_{v}\right|$, defined by the small angle $\Delta \theta_{k}$ subtended by phonon wave vector $\mathbf{k}_{\|}$within the surface divided by the corresponding angle $\Delta \theta_{v}$ spanned by the group velocity $\mathbf{v}$. Based on the Green's tensor approach Camley and Maradudin also studied the focusing of both RSW and pseudosurface waves (PSW) propagating on the surfaces of several cubic crystals. ${ }^{7}$ Their formulation is similar to that of Maris ${ }^{3}$ for bulk phonons and the stationary phase analysis is developed. In effect, they plotted the caustics directions where the focusing factor $A$ diverges. Such directions exist, in general, for both RSW and PSW in a solid.

Recently, Kolomenskii and Maznev ${ }^{8}$ have generated surface acoustic waves by the irradiation of pulsed laser on the surfaces of several cubic crystals. Using an interesting technique called surface-wave induced dust particle removal from a surface they have observed that the strong focusing of surface waves does occur as predicted. The surface waves detected in their experiment have a characteristic frequency of about $50 \mathrm{MHz}$, or the corresponding wavelength is about $0.1 \mathrm{~mm}$, so the interference of the waves involved would be important in interpreting 
the details of their experimental results.

The purpose of the present work is to study theoretically the effect of finite wavelength on the ballistic propagation of both RSW and PSW on anisotropic crystal surfaces. We will show that a feature of the experiment by Kolomenskii and Maznev, ${ }^{8}$ which has not been explained by the simple argument based on the ray picture can be understood in the framework of our formulation.

\section{FORMULATION}

Asymptotic expressions for the amplitudes of surface waves have been derived by Shirasaki and Makimoto, ${ }^{9}$ and also by Camley and Maradudin ${ }^{7}$ based on the stationary-phase analysis of the Green's tensor for a semiinfinite anisotropic (piezoelectric) elastic medium. In the present study we try to obtain the displacement amplitude $\mathbf{u}(\mathbf{r}, t)$ of surface waves by considering the Lamb's problem $^{10}$ for an anisotropic elastic continuum occupying $z\left(=x_{3}\right)>0$ with surface at $z=0$. The equations we should solve are

$\rho \omega^{2} u_{i}+c_{i j m n} \partial_{j} \partial_{n} u_{m}=0$ for $z>0$

$\sigma_{3 j} \equiv c_{3 j m n} \partial_{n} u_{m}=s_{j} \delta\left(\mathbf{x}_{\|}\right) e^{-i \omega t} \quad$ for $z=0$

where $\rho$ is the mass density of the crystal, $c_{i j m n}$ is the elastic-constant tensor, $\sigma_{i j}$ is the stress tensor, and $\mathbf{r}=\left(\mathbf{x}_{\|}, z\right)\left[\mathbf{x}_{\|}=\left(x_{1}, x_{2}\right)\right.$ is the vector on the surface $]$. The summation convention over repeated indices is implied. We assume that the normal component of the stress oscillating with frequency $\omega$ is acting at the origin $\mathbf{x}_{\|}=\mathbf{0}$ on the surface, i.e., $s_{j}=s_{0} \delta_{3 j}$ and $s_{0}$ is a constant. This boundary condition should approximately be applicable, for instance, to the excitation of surface waves by a focused ultrasound beam emitted from an immersion transducer. ${ }^{5}$

The solution of Eq. (1) takes the form

$$
\begin{aligned}
\mathbf{u}(\mathbf{r}, t)= & \frac{1}{(2 \pi)^{2}} \int d^{2} k_{\|} e^{i\left(\mathbf{k}_{\|} \cdot \mathbf{x}_{\|}-\omega t\right)} \\
& \times \sum_{m=1}^{3} K^{(m)} \epsilon^{(m)} e^{i k_{z}^{(m)} z}
\end{aligned}
$$

where $\mathbf{k}=\left(\mathbf{k}_{\|}, k_{3}\right)=\left(\mathbf{k}_{\|}, k_{z}^{(m)}\right)$ and the components of the wave vector perpendicular to the surface $k_{z}^{(m)}\left(\operatorname{Im}\left[k_{z}^{(m)}\right]>0, m=1,2,3\right)$ are the solutions of

$$
\operatorname{det}\left(L_{i l}\right)=0
$$

for a given $\mathbf{k}_{\|}$with

$$
L_{i l}=-\rho \omega^{2} \delta_{i l}+c_{i j l n} k_{j} k_{n}
$$

and $\epsilon^{(m)}$ are the polarization vectors satisfying

$$
L_{i l}(\mathbf{k}, \omega) \epsilon_{l}=0
$$

with $k_{3}=k_{z}^{(m)}$. In Eq. (3) $K^{(m)}$ are the weighting fac- tors determined from the boundary condition Eq. (2). Explicitly, for the (001) surface of a cubic crystal

$$
\begin{array}{r}
c_{44} \sum_{m}\left[k_{i} \epsilon_{3}^{(m)}+k_{z}^{(m)} \epsilon_{i}^{(m)}\right] K^{(m)} \equiv \sum_{m} W_{i}^{(m)} K^{(m)}=0 \\
(i=1,2),
\end{array}
$$

$$
\begin{aligned}
\sum_{m}\left\{c_{12}\left[k_{1} \epsilon_{1}^{(m)}+k_{2} \epsilon_{2}^{(m)}\right]+c_{11} k_{z}^{(m)} \epsilon_{3}^{(m)}\right\} K^{(m)} & \\
& \equiv \sum_{m} W_{3}^{(m)} K^{(m)}=s_{0} .
\end{aligned}
$$

Solving Eqs. (7) and (8), we find

$$
K^{(m)}=s_{0} h^{(m)} / W,
$$

with $W=\operatorname{det}\left(W_{i}^{(m)}\right)$ and

$$
h^{(m)}=W_{1}^{(m+1)} W_{2}^{(m+2)}-W_{2}^{(m+1)} W_{1}^{(m+2)},
$$

where the superscripts are defined modulo 3 , e.g., $W_{i}^{(4)}=$ $W_{i}^{(1)}$. Thus, Eq. (3) becomes

$$
\begin{aligned}
\mathbf{u}(\mathbf{r}, t)= & \frac{s_{0} e^{-i \omega t}}{(2 \pi)^{2}} \int d k_{\perp} \int d k_{\|} \frac{e^{i k_{\|} x_{\|}}}{W\left(\mathbf{k}_{\|}, \omega\right)} \\
& \times \sum_{m} h^{(m)} \epsilon^{(m)} e^{i k_{z}^{(m)} z}
\end{aligned}
$$

where $x_{\|}=\left|\mathbf{x}_{\|}\right|$. In deriving Eq. (11), we have rotated the coordinate system so that $k_{\|}$direction is parallel to the vector $\mathbf{x}_{\|}$and $k_{\perp}$ direction normal to $\mathbf{x}_{\|}$within the surface. Here we note that $W=0$ defines the dispersion relation of surface waves. It is known that for a fixed $\omega$, $W=0$ has a simple zero at a real $\mathbf{k}_{\|}$. This corresponds to RSW. For PSW, however, a complex $\mathbf{k}_{\|}$satisfies $W=0$ for a real $\omega$ (also one of $k_{z}^{(m)}$ has a negative imaginary part). So, for the moment, we consider the case of RSW.

Now, replacing $\omega$ by $\omega+i \eta$ ( $\eta$ is an infinitesimal positive number), we perform the integral with respect to $k_{\|}$ so that Eq. (11) may represent outgoing waves. Thus, putting $z=0$, we find the displacement vector at the surface

$\mathbf{u}\left(\mathbf{x}_{\|}, t\right)=\frac{i s_{0}}{2 \pi} e^{-i \omega t} \int_{W=0} d k_{\perp} \frac{e^{i k_{\|} x_{\|}}}{\partial W / \partial k_{\|}} \sum_{m} h^{(m)} \epsilon^{(m)}$,

where $k_{\|}$is a function of $k_{\perp}$ and $\omega$, i.e., $k_{\|}=k_{\|}\left(k_{\perp}, \omega\right)$, and the integral should be done over the slowness curve in the $\mathbf{k}_{\|}$plane defined by $W\left(\mathbf{k}_{\|}, \omega\right)=0$. The prime means that the integral is restricted to the portion of the slowness curve for which the component of the groupvelocity vector projected to the $\mathbf{x}_{\|}$direction is positive.

For PSW, $W=0$ is satisfied for a complex $\mathbf{k}_{\|}$as remarked above. However, the attenuation rate is small, i.e., $\operatorname{Im}\left[\mathbf{k}_{\|}\right] / \operatorname{Re}\left[\mathbf{k}_{\|}\right]=10^{-3}-10^{-5}$ (see Fig. 6, below), 
so we expect that Eq. (12) would also be used for PSW as far as the propagation distance of $1-\mathrm{cm}$ and $10-\mathrm{MHz}$ frequency range are concerned $\left(\operatorname{Re}\left[k_{\|}\right] x_{\|} \sim 100\right.$ and $\operatorname{Im}\left[k_{\|}\right] x_{\|}<10^{-1}$ ).

For $x_{\|}$much larger than the wavelength of surface waves on the given slowness curve we can further carry out the integral of Eq. (12) by applying the method of stationary phase. Following the procedures similar to those developed by Shirasaki and Makimoto, ${ }^{9}$ and also by Camley and Maradudin, ${ }^{7}$ we obtain

$$
\begin{aligned}
\mathbf{u}\left(\mathbf{x}_{\|}, t\right)= & \frac{i s_{0} e^{-i \omega t}}{\left(2 \pi x_{\|}\right)^{1 / 2}} \sum_{\alpha} \frac{e^{i\left(\mathbf{k}_{\|} \cdot \mathbf{x}_{\|}+\pi / 4-\phi / 2\right)}}{(|\nabla W \| a|)^{1 / 2}} \\
& \times\left.\sum_{m} h^{(m)} \epsilon^{(m)}\right|_{\mathbf{k}_{\|}=\mathbf{k}_{\|}^{(\alpha)}},
\end{aligned}
$$

where $\nabla$ is the gradient with respect to $\mathbf{k}_{\|}, a$ $=t_{i} t_{j} \nabla_{i} \nabla_{j} W, \mathbf{t}=\left(-\nabla_{1} W, \nabla_{2} W\right) /|\nabla W|$ is the unit vector tangent to the slowness curve, $\phi$ is the phase of $a$, and the summation over $\alpha$ is taken over the wave vectors $\mathbf{k}_{\|}=\mathbf{k}_{\|}^{(\alpha)}$ on the slowness curve for which the outward normals of the slowness curve are parallel to $\mathbf{x}_{\|}$. Equation (13) corresponds to the geometrical acoustics approximation for the displacement vector, which has been derived by assuming $a \neq 0$. Apparently $a$ is proportional to the curvature $\kappa$ of the slowness curve defined by $\kappa=t_{i} t_{j} \nabla_{i} \nabla_{j} W /|\nabla W|$ and vanishes at the inflection points, giving rise to the caustic directions in the real space where the geometrical acoustics approximation is no longer valid. The corrections to Eq. (13) at the points satisfying $a=0$ are discussed in Refs. 7 and 9 .

\section{NUMERICAL RESULTS}

Figure 1 plots the phase velocities $c$ of RSW and PSW on the (001) plane of silicon together with the sound velocity of bulk slow transverse (ST) waves. We see the the degeneracies of RSW and PSW with ST branch near the [110] direction and at an angle about $22^{\circ}$ from the [100] direction, respectively. The slowness curves defined by $\left|\mathbf{k}_{\|}\right|=\omega / c\left(\mathbf{k}_{\|}\right)$( $\omega$ is a given frequency) in the wave vector space and the corresponding group velocity curves in the real space are also given in Figs. 2(a) and 2(b) for both RSW and PSW. The group-velocity curves are plotted by dots which represent the distributions of groupvelocity vectors calculated by assuming a uniform angular distribution of wave vectors. This means that the concentrations of dots on the group-velocity curves measure the focusing and defocusing of the surface waves at a specified propagation direction.

In the geometrical acoustics approximation the quantitative understanding of the focusing of surface waves will be gained by calculating the focusing factor $A$. The focusing factor describes the enhancement in phonon flux in a given direction to that in an isotropic solid and is a quantity measurable in the heat pulse experiment for bulk phonons. ${ }^{1}$ Here we note that the focusing factor is also related to the curvature of the slowness curve as

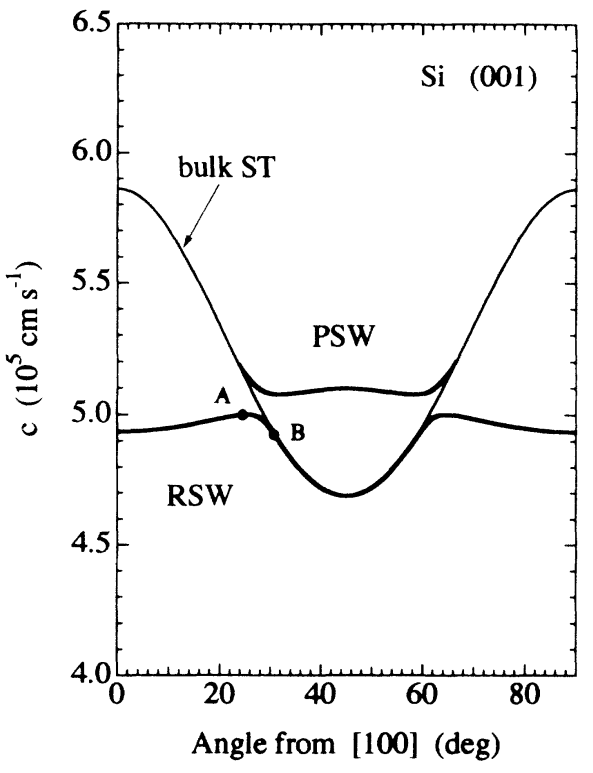

FIG. 1. Angular $\left(\theta_{k}\right)$ dependences of the phase velocities of RSW, PSW (bold lines), and bulk ST phonons (thin line) in the (001) plane of silicon. For points $A$ and $B$, see the caption of Fig. 2.

$A^{-1}=\left|\kappa \omega v / c^{2}\right|$, where $v=|\mathbf{v}|$. Thus, the cusps on the group velocity curve of the Rayleigh branch $\left[A^{\prime}\right.$ and $B^{\prime}$ in Fig. 2(b)] which originate from the inflection points on the slowness curve $[A$ and $B$ in Fig. 2(a)] define the caustic directions at which the focusing factor becomes infinite. In the pseudosurface branch no such cusps ap-
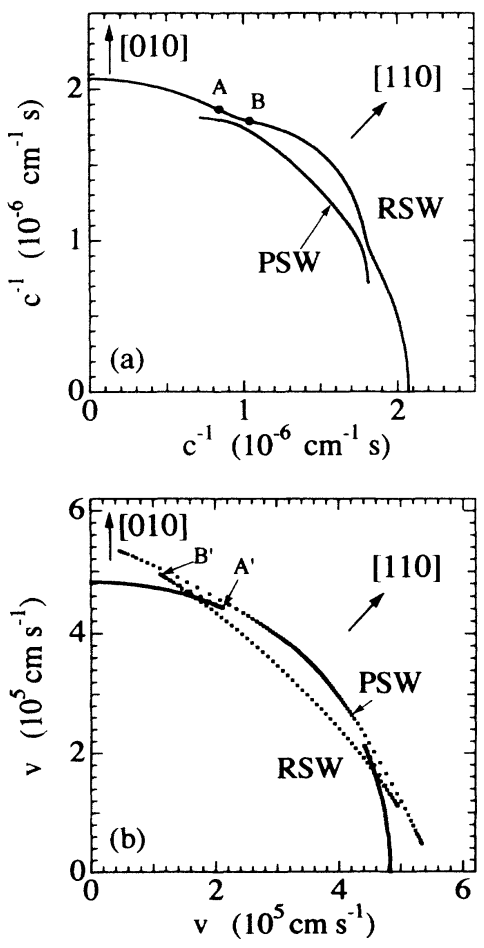

FIG. 2. (a) Slowness curves and (b) group-velocity curves of RSW and PSW on the (001) plane of silicon. The points $A$ and $B$ are the inflection points on the slowness curve of the Rayleigh branch and $A^{\prime}$ and $B^{\prime}$ are the corresponding points on the group-velocity curve defining the caustic directions. 
pear on the (001) plane of silicon but they do appear on the same crystal plane of other crystals. ${ }^{7}$ Figure 3 plots the angular dependences of the focusing factors of both RSW and PSW on the (001) plane of silicon. The focusing factors of this figure have been calculated numerically according to the definition $\left|\Delta \theta_{k} / \Delta \theta_{v}\right|$ with $\Delta \theta_{k}=0.01^{\circ}$ and $\Delta \theta_{v}=0.5^{\circ}$, so the divergence of $A$ in a caustic direction is suppressed by the finite resolution for the angle $\theta_{v}$. We see that RSW focus strongly in the region between the caustics located at $\theta_{v}=12.9^{\circ}\left(B^{\prime}\right)$ and $25.7^{\circ}\left(A^{\prime}\right)$ from the [100] axis but PSW focus rather gently in the [110] direction.

In a typical experiment on the propagation of ultrasonic bulk waves, the distance $r=|\mathbf{r}|(\simeq 1 \mathrm{~cm})$ between the source and the detector is considerably larger than their wavelength $\lambda(\simeq 0.3 \mathrm{~mm}$ at $20 \mathrm{MHz})$. This suggests that the method of stationary phase should be a good approximation to evaluate the displacement vector $\mathbf{u}$ associated with the acoustic field excited with a broad distribution of wave vector $\mathbf{k}$. The asymptotic form of the displacement field for large $r$ was obtained by Maris for bulk phonons. ${ }^{3}$ In the work of Maris, the prefactors of $e^{i \mathbf{k} \cdot \mathbf{r}}$ in the expression of the displacement vector [similar to Eq. (12)] are approximated to be constant and he discussed the phonon intensity at $\mathbf{r}$ in terms of

$$
I(\mathbf{r})=\left|\int_{\mathbf{k}_{0}} d^{2} k e^{i \mathbf{k} \cdot \mathbf{r}}\right|^{2}
$$

where the integral should be taken over the region of the slowness surface around $\mathbf{k}_{0}$ satisfying $\mathbf{v}\left(\mathbf{k}_{\mathbf{0}}\right) \| \mathbf{r}$. Applying the similar idea to the surface wave propagation, we tentatively consider the corresponding quantity

$$
I\left(\mathbf{x}_{\|}\right)=\left|\int_{W=0}^{\prime} d k_{\perp} e^{i k_{\|} \mathbf{x}_{\|}}\right|^{2} .
$$

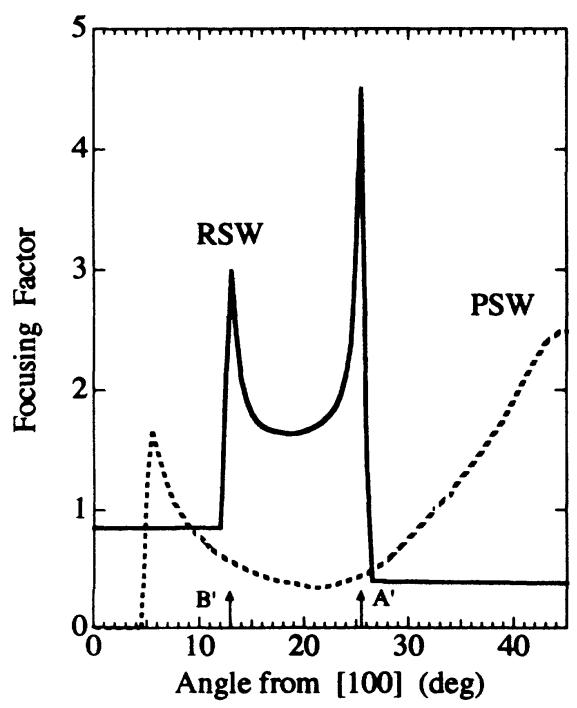

FIG. 3. Angular $\left(\theta_{v}\right)$ dependences of the focusing factors of RSW (solid line) and PSW (dashed line) on the (001) plane of silicon. $A^{\prime}$ and $B^{\prime}$ are the caustic directions shown in Fig. 2(b).
This intensity is obtained by leaving out from Eq. (12) the residue function at the pole of the surface wave, i.e., we assume that the residue function is insensitive to $\mathbf{k}_{\|}$as remarked by Weaver and co-workers ${ }^{5}$ for the bulk waves. In Figs. 4 and 5 we have plotted the numerical calculations of Eq. (15) for both RSW and PSW. The propagation distance $\left(x_{\|}\right)$is assumed to be $1 \mathrm{~cm}$. At $100-\mathrm{MHz}$ frequency the averaged behavior of $I\left(\mathbf{x}_{\|}\right)$exhibits the similar angular dependence as the focusing factor. At $20-\mathrm{MHz}$ frequency, however, the oscillations of $I\left(\mathbf{x}_{\|}\right)$due to the interference is very significant, though $I\left(\mathbf{x}_{\|}\right)$has still large amplitudes in the focusing regions. Here, we note that the dotted lines of Fig. 5 are $I\left(\mathbf{x}_{\|}\right)$'s for PSW obtained by neglecting $\operatorname{Im}\left[\mathbf{k}_{\|}\right]$, but the bold solid lines include finite values of $\operatorname{Im}\left[\mathbf{k}_{\|}\right]$in the calculation of $I\left(\mathbf{x}_{\|}\right)$. As shown in Fig. $6 \operatorname{Im}\left[\mathbf{k}_{\|}\right] / \operatorname{Re}\left[\mathbf{k}_{\|}\right]$takes rather large values $\left(>10^{-3}\right)$ at angles $\theta_{k}$ smaller than $30^{\circ}$. This causes the attenuation of $I\left(\mathbf{x}_{\|}\right)$at angles $\theta_{v}$ also smaller than $30^{\circ}$.

At a finite phonon frequency $I\left(\mathbf{x}_{\|}\right)$defined by Eq. (15) exhibits a good correspondence to the focusing factor as
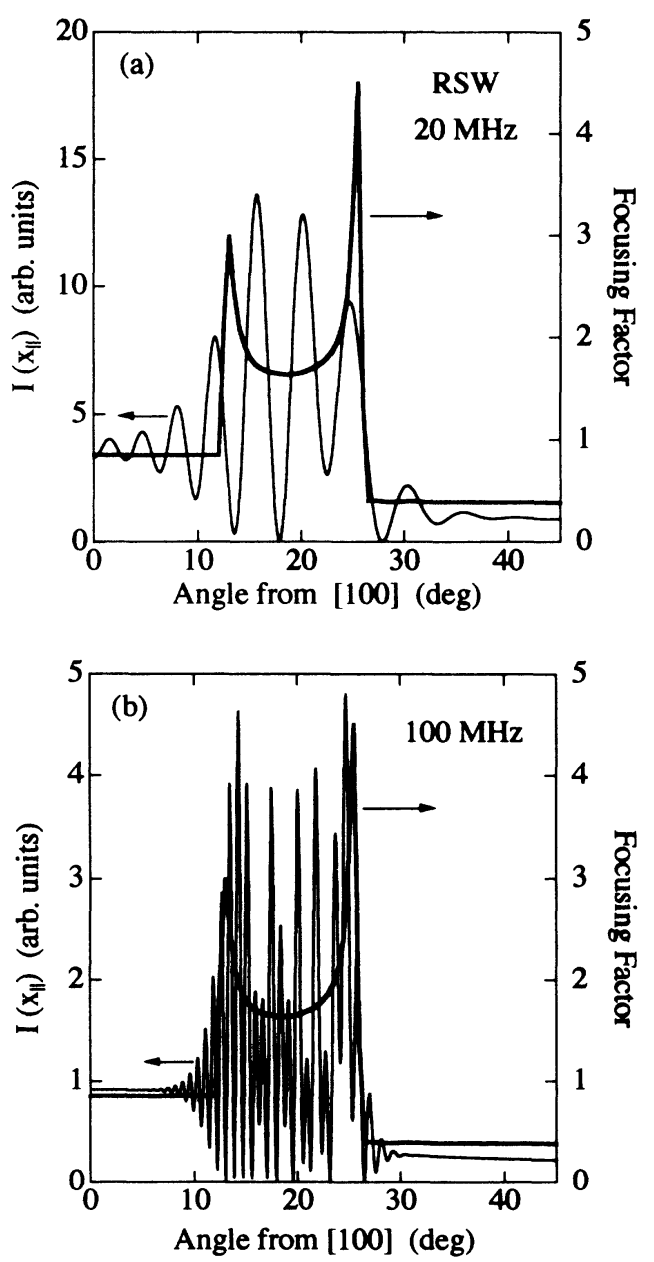

FIG. 4. Angular dependences of the intensity $I\left(\mathbf{x}_{\|}\right)$[Eq. (15)] for RSW (thin lines) together with the focusing factor (bold lines) in the (001) plane of silicon. The propagation distance $x_{\|}$is $1 \mathrm{~cm}$ and the frequencies are (a) $20 \mathrm{MHz}$, (b) $100 \mathrm{MHz}$. 


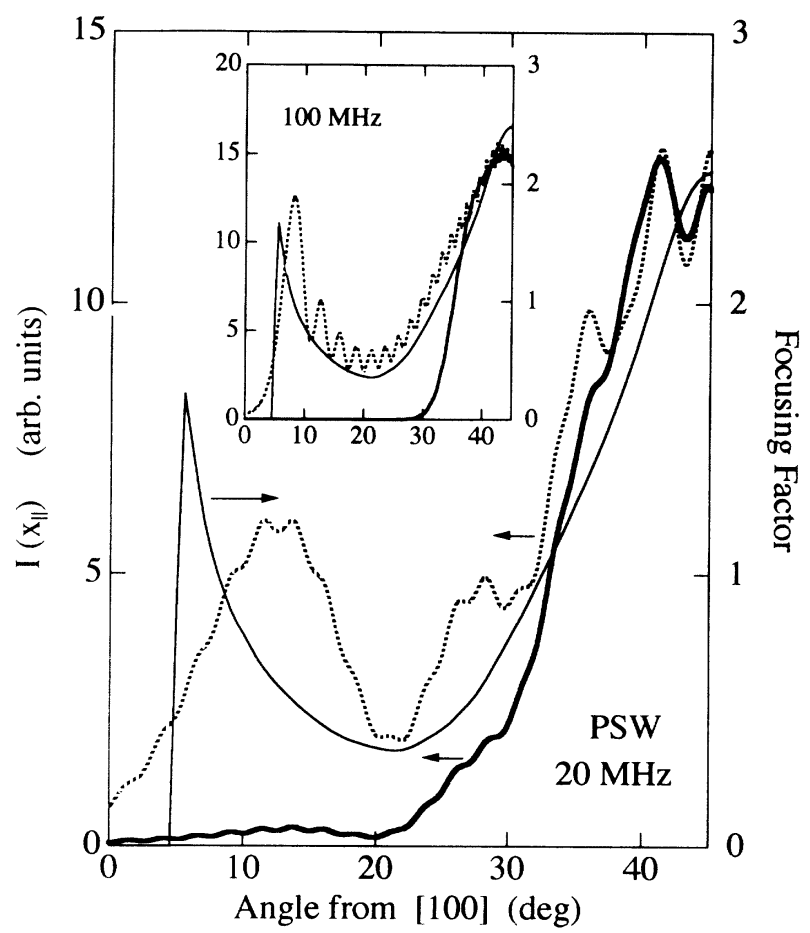

FIG. 5. Angular dependences of the intensity $I\left(\mathbf{x}_{\|}\right)$[Eq. (15)] for PSW with $\operatorname{Im}\left[\mathbf{k}_{\|}\right]$(bold line) and without $\operatorname{Im}\left[\mathbf{k}_{\|}\right]$ (dotted line) together with the focusing factor (thin solid line) in the (001) plane of silicon. The propagation distance $x_{\|}$is $1 \mathrm{~cm}$ and the frequency is $20 \mathrm{MHz}$. Inset is the same plot for $100 \mathrm{MHz}$.

shown above. So, $I\left(\mathbf{x}_{\|}\right)$may be regarded as a quantity which measures the focusing of surface phonons or surface waves at finite frequencies. However, in an experiment utilizing the coherent ultrasonic beams the detected intensity of acoustic fields would be quite sensitive to the polarization vectors involved. This must especially be the case for the surface wave experiments conducted with a sample immersed in the water. Here it should be noted

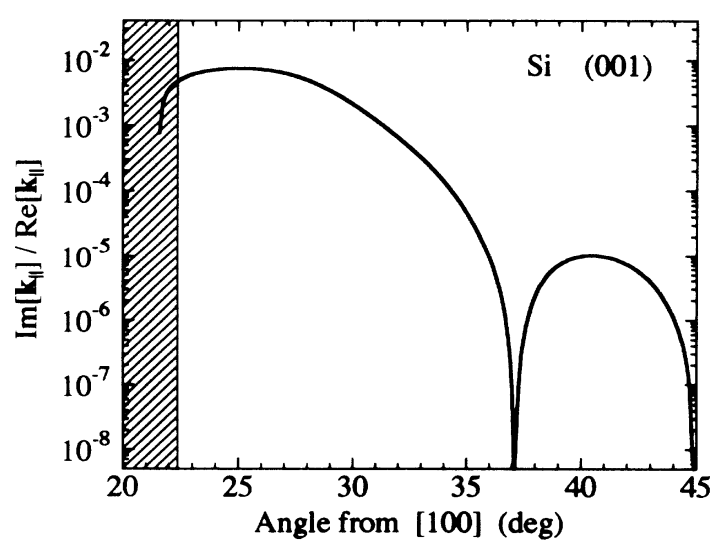

FIG. 6. Angular $\left(\theta_{k}\right)$ dependence of $\operatorname{Im}\left[\mathbf{k}_{\|}\right]$for PSW in the (001) plane of silicon. In the hatched region PSW degenerate into bulk ST branch. the bulk phonons in solids consist of three independent modes propagating with different velocities. The surface acoustic waves are also comprised of three vibrations polarized nearly perpendicular to each other but they travel with the same velocity and their relative amplitudes are settled by the boundary condition at the surface. Thus, the exact calculation of Eq. (12) for the angular dependence of $\left|u_{i}\right|^{2}$ should be very important to quantify the intensity of surface waves.

Taking explicit account of the prefactor of the exponential function $e^{i k_{\|} x_{\|}}$of Eq. (12), we plot in Figs. 7(a) and 7 (b) the angular dependences of $\left|u_{z}\right|^{2},\left|u_{\|}\right|^{2}$, and $\left|u_{\perp}\right|^{2}$ for RSW on the (001) surface of silicon $\left(u_{\|}\right.$and $u_{\perp}$ are the components of the displacement vector parallel and perpendicular to $\mathbf{x}_{\|}$within the surface). The similar plots for PSW are given in Fig. 8. (The parameters assumed are the same as Fig. 4.) In Fig. 7, the corresponding quantities calculated from the asymptotic formula Eq. (13) valid at high-frequency limit are also plotted by dashed lines for comparison. The angular dependences of $\left|u_{z}\right|^{2}$ and $\left|u_{\|}\right|^{2}$ for RSW are quantitatively quite different from Figs. 3 and 4 . The large enhancement observed in the fo-
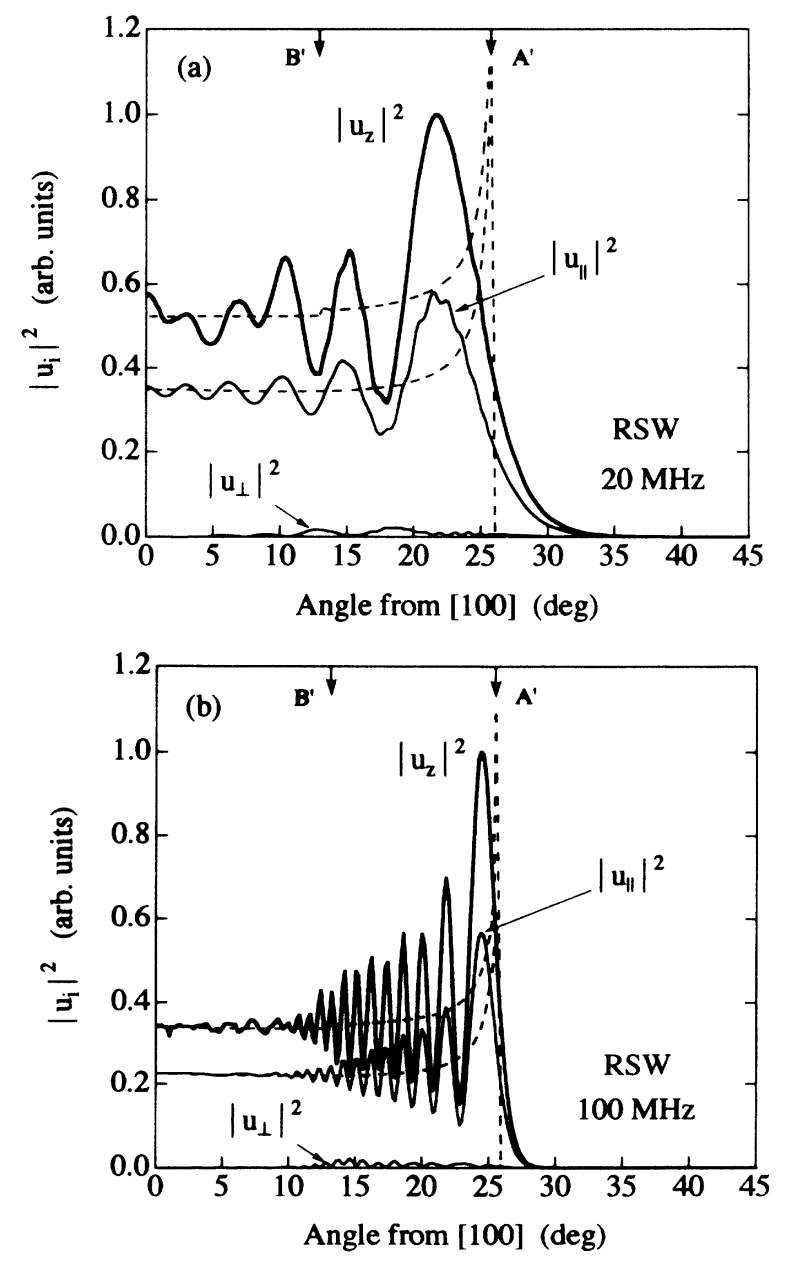

FIG. 7. Squared amplitudes of the lattice displacements of RSW in the (001) surface of silicon. Dashed lines are the asymptotic expressions obtained from Eq. (13). The propagation distance $x_{\|}$is $1 \mathrm{~cm}$ and the frequencies chosen are (a) $20 \mathrm{MHz}$ and (b) $100 \mathrm{MHz}$. 


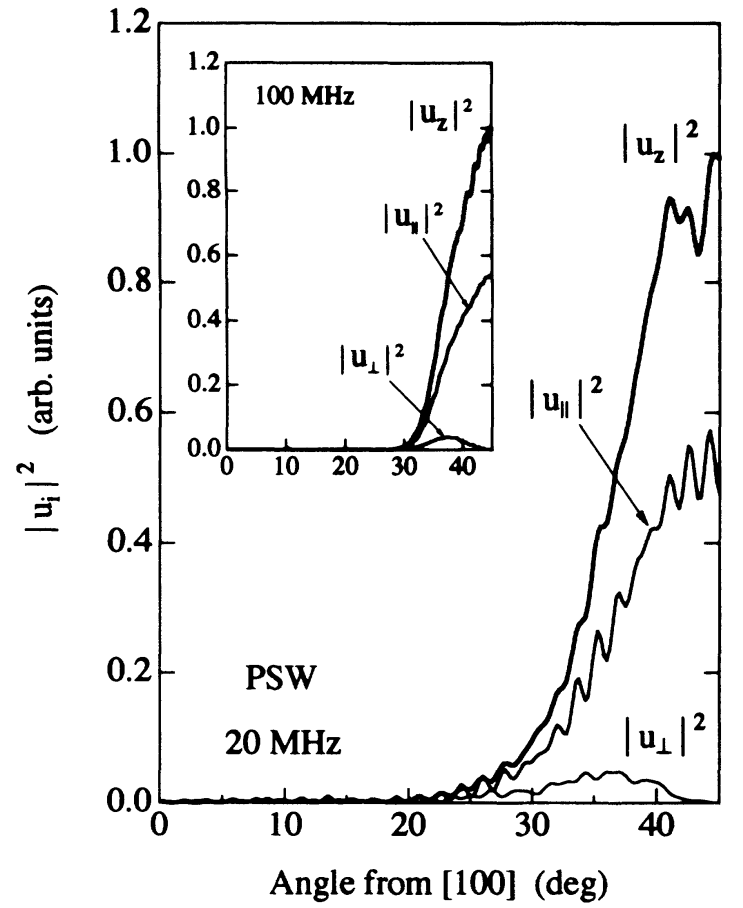

FIG. 8. Squared amplitudes of the lattice displacements of PSW in the (001) surface of silicon. The propagation distance $x_{\|}$is $1 \mathrm{~cm}$ and the frequency is $20 \mathrm{MHz}$. The inset shows the same plot for $100 \mathrm{MHz}$.

cusing factor at $\theta_{v} \simeq 13^{\circ}\left(B^{\prime}\right)$ is not seen in $\left|u_{i}\right|^{2}$ of Fig. 7 though the enhancements at $\theta_{v} \simeq 26^{\circ}\left(A^{\prime}\right)$ are present for $\left|u_{\|}\right|^{2}$ and $\left|u_{z}\right|^{2}$. Also, the amplitudes at the surface are practically zero at angles larger than $30^{\circ}$. These results imply that the residue function in Eq. (12) is crucial in the evaluation of the displacement amplitudes. The absence of the enhancement at $\theta_{v} \simeq 13^{\circ}$ is understood by the fact that the cusp $B^{\prime}$ seen in Fig. 2(b) comes from the inflection point on the slowness curve at $\theta_{k} \simeq 30.5^{\circ}[B$ in Fig. 2(a)]. The displacement vector of RSW at $\theta_{k} \geq 30^{\circ}$ is polarized almost within the surface and perpendicular to the wave vector, i.e., RSW are the shear waves with horizontal polarization in character, and hence $u_{z}$ and $u_{\|}$ are little excited around this direction.

Here, it is interesting to note that the asymptotic forms of the squared amplitudes calculated from Eq. (13) for RSW (averaged over frequency) well describe the averaged angular dependences of those obtained from Eq. (12). Equation (13) vanishes at angles satisfying $a=0$, i.e., at the caustic directions $A^{\prime}$ and $B^{\prime}$ on the (001) silicon. Both $\left|u_{z}\right|^{2}$ and $\left|u_{\|}\right|^{2}$ calculated numerically from Eq. (13) indeed exhibit the sharp elevations at the direction $A^{\prime}$ (and practically zero at larger angles), but no clear enhancements can be seen at the direction $B^{\prime}$ within the resolution of the present calculations. The latter is again due to the nature of the polarization of RSW at angle $\theta_{k}$ near the [110] direction. In addition, for $\theta_{k}>30^{\circ}$ the magnitude of the surface displacement excited by the stress at $\mathbf{x}_{\|}=0$ is very small. (More explicitly, the residue of $K^{(m)}$ [Eq. (9)] at the pole of RSW becomes small.) This result is superimposed to the above effect, leading to the suppression of RSW amplitudes at the direction $B^{\prime}$ (and also at $\theta_{v}>26^{\circ}$ ).

For PSW the squared amplitudes are very small at $\theta_{v} \leq 30^{\circ}\left(\theta_{k} \leq 27^{\circ}\right)$. One of the reasons has already been described in the explanation of Fig. 5. The second reason is that PSW also degenerate into the bulk ST waves at $\theta_{k} \simeq 22^{\circ}$, so the surface displacement is small at these angles. Note that the asymptotic expression Eq. (13) vanishes for PSW except at two angles where PSW become genuine surface waves $\left(\operatorname{Im}\left[\mathbf{k}_{\|}\right]=0\right)$, i.e., $[110]$ direction and $\theta_{v}=39^{\circ}\left(\theta_{k} \simeq 37^{\circ}\right)$ for the (001) silicon (see Fig. 6).

The acoustic Poynting vector defined by $P_{i}=$ $-\operatorname{Re}\left[\sigma_{i j} \dot{u}_{j}^{*}\right] / 2$ should be another important quantity to measure the focusing of surface waves. For both RSW and PSW the component $P_{3}$ normal to the surface vanishes identically at the surface for $\mathbf{x}_{\|} \neq 0$. This is because $\sigma_{3 j}=0$ due to the boundary condition Eq. (2). The only sizable component is $P_{\|}$, i.e., the component parallel to $\mathbf{x}_{\|}$and the component $P_{\perp}$ perpendicular to $\mathbf{x}_{\|}$is small. We find the angular dependence of $P_{\|}$for RSW is quite similar to those of $\left|u_{z}\right|^{2}$ and $\left|u_{\|}\right|^{2}$ shown in Fig. 7.

\section{CONCLUDING REMARKS}

After the observation of ballistic phonon focusing in the bulk of crystals, many groups tried to detect the similar effect at crystal surfaces. Unfortunately, however, no positive result on the effect has been reported until recently. One of the reasons is that they used the heat pulse method to excite phonons emanating from a point source at low temperatures. The dominant phonon frequencies excited in their experiments are typically $100 \mathrm{GHz}$ or higher, and the mean free path against surface-roughness scattering $\left(\propto \omega^{-5}\right)$ is very short, ${ }^{11}$ making the ballistic propagation of such surface heat pulses over macroscopic distances impossible.

In the traditional acoustics, ultrasonic surface waves of frequencies lower than $1 \mathrm{GHz}$ are rather easily generated electromechanically, but point sources were not readily available. This drawback has been overcome by the techniques using focused ultrasonic beams ${ }^{5}$ or laser pulses. ${ }^{4,8}$ The recent experiment by Kolomenskii and Maznev ${ }^{8}$ with laser excited surface waves has observed very clearly the existence of the directions along which the strong focusing occurs at crystal surfaces. Those directions are, for the most part, consistent with the theoretical predictions for the caustics of surface phonons obtained by geometrical acoustics. For the (001) surface of silicon, they did see the focusing of RSW at $\theta_{v} \simeq 26^{\circ}$ (the direction $A^{\prime}$ ) but no evidence of the strong focusing was obtained at $\theta_{v} \simeq 13^{\circ}$ (the direction $B^{\prime}$ ). Their experiment is sensitive to the lattice displacement normal to the surface and Fig. 7 which we have calculated for the RSW amplitudes also exhibits the absence of the effect at the latter angle. More detailed measurements of the angular dependence of the surface wave focusing at ultrasonic frequencies would reveal the finite wavelength effect as predicted in this work.

In the present study, we have assumed that the solid 
surface is in contact with vacuum, so it is free from the stress except at the origin. However, as mentioned repeatedly, the technique for the generation of focused coherent ultrasound beams on the sample immersed in liquid will be applied to the excitation of surface acoustic waves and also to the study of the interference effect on the phonon focusing at surfaces. In this respect the formulation taking account of the loading of the free surface with liquid should be necessary. In particular, it has been remarked that the characteristics of PSW are sensitive to the presence of liquid at solid surfaces. ${ }^{12}$

\section{ACKNOWLEDGMENTS}

One of the authors (S.T.) has benefited from conversations with J. P. Wolfe, M. Hauser, and R. Vines. This work was supported in part by the Ogasawara Foundation for the Promotion of Science and Engineering.
${ }^{1}$ B. Taylor, H. J. Maris, and C. Elbaum, Phys. Rev. Lett. 23, 416 (1969); Phys. Rev. B 3, 1462 (1971).

${ }^{2}$ See, for a review, G. A. Northrop and J. P. Wolfe, in Nonequilibrium Phonon Dynamics, edited by W. E. Bron (Plenum, New York, 1985).

${ }^{3}$ H. J. Maris, Phys. Rev. B 28, 7033 (1983).

${ }^{4}$ A. G. Every, W. Sachse, K. Y. Kim, and M. O. Thompson, Phys. Rev. Lett. 65, 1446 (1990); A. G. Every and W. Sachse, Phys. Rev. B 44, 6689 (1991).

${ }^{5}$ M. R. Hauser, R. L. Weaver, and J. P. Wolfe, Phys. Rev. Lett. 68, 2604 (1992); R. L. Weaver, M. R. Hauser, and J. P. Wolfe, Z. Phys. B 90, 27 (1993).

${ }^{6} \mathrm{~S}$. Tamura and K. Honjo, Jpn. J. Appl. Phys. Suppl. 3 20, 17 (1980); K. Honjo and S. Tamura (unpublished).
${ }^{7}$ R. E. Camley and A. A. Maradudin, Phys. Rev. B 27, 1959 (1983).

8 Al. A. Kolomenskii and A. A. Maznev, Pis'ma Zh. Eksp. Teor. Fiz. 53, 403 (1991) [JETP Lett. 53, 423 (1991)]; Phys. Rev. B 48, 14502 (1993).

${ }^{9}$ H. Shirasaki and T. Makimoto, J. Appl. Phys. 49, 658 (1978); 49, 661 (1978); 50, 2795 (1979).

${ }^{10}$ See, for example, W. M. Ewing, W. S. Jardetzky, and F. Press, Elastic Waves in Layered Media (McGraw-Hill, New York, 1957).

${ }^{11}$ A. A. Maradudin and D. L. Mills, Ann. Phys. (N. Y.) 100, 262 (1976).

${ }^{12}$ T. C. Lim and G. W. Farnell, J. Acous. Soc. Am. 45, 845 (1969). 